\title{
Strategic top-down control versus attentional bias by previous reward history
}

\author{
Jennifer Lynn ${ }^{1}$ • Myoungju Shin ${ }^{1}$
}

Published online: 4 June 2015

(C) The Psychonomic Society, Inc. 2015

\begin{abstract}
Rewards modify performance so that attentional priority is given to stimuli associated with a higher probability of reward. A stimulus associated with reward attracts attention even when it is no longer relevant. In this study, we explored whether or not strategic top-down control can be employed to overcome the attentional bias from a recent reward-stimulus association. Four groups of 12 participants completed a spatial-cueing task involving two phases, in which the cue associated with the target location changed from Phase 1 to Phase 2. Attentional-bias effects toward a previously rewarded cue were demonstrated when the rewarded cue from Phase 1 interfered with the orienting toward a nonrewarded but valid cue in Phase 2. Associating the Phase 2 cue with a higher reward than had been used in Phase 1 resulted in a rapid orientation of attention to the new cue. These findings suggest that pathologies characterized by maladaptive attentional biases (e.g., addiction) may be counteracted by treatments that manipulate motivation by enhancing the subjective relevance of rewards that are less harmful.
\end{abstract}

Keywords Attentional capture $\cdot$ Attention $\cdot$ Reward history . Motivation

Recent studies have shown that attentional control is directly regulated by primary reward (e.g., food and sexual stimuli) and secondary reward (e.g., money; Awh, Belopolsky, \& Theeuwes, 2012; Chelazzi, Perlato, Santandrea, \& Della Libera, 2013). Particularly, financial reward enhances goal-

Myoungju Shin

jshin@csu.edu.au

1 School of Psychology, Charles Sturt University, Panorama Avenue, Bathurst, NSW 2795, Australia directed control by motivation in human participants, strengthening the focus and stability of the current goal and fine-tuning the attentional resources available. Reward boosts perceptual process capacity, enhancing detection sensitivity in a visually challenging attention task (Engelmann, Damaraju, Padmala, \& Pessoa, 2009). Reward also enhances executive control in a variety of domains, including selective attention (Gable \& Harmon-Jones, 2008), response inhibition (Verbruggen \& De Houwer, 2007), and working memory (Gray, 2001). Reward anticipation is associated with performance gain in response speed and accuracy, without any speed-accuracy trade-offs (Bijleveld, Custers, \& Aarts, 2010; Savine, Beck, Edwards, Chiew, \& Braver, 2010). Although pairing target stimuli with reward may be beneficial to an individual in many circumstances, recent work has highlighted that it may also lead to undesired aftereffects, whereby a stimulus previously associated with reward attracts attention even when it would be more beneficial to ignore it. Our study is concerned with manipulating motivation to determine whether strategic top-down control can be employed to overcome the ongoing effects of recent reward history that are not conducive to ongoing optimal task performance.

Beneficial effects of motivation by reward in selective attention have been investigated using various experimental paradigms. Savine et al. (2010) examined the effect of motivation on cognitive control, using a switching task in which participants received a reward based on their performance. Faster response times (RTs; by $\sim 250 \mathrm{~ms}$ ) and fewer errors were observed for trials within rewarded blocks, without any speed-accuracy trade-offs, and the facilitation was significantly stronger for mixed blocks (i.e., task-switching block) with high cognitive demands than for single-task blocks with low cognitive loads. Similar results were observed by Veling and Aarts (2010) using a Stroop task. Reward was shown to reduce the Stroop interference (i.e., participants take longer 
naming the color of a word if the color and meaning of the word are incongruent) typically observed in the task. In particular, Engelmann et al. (2009) investigated the effect of motivation in a Posner cue-target task similar to the one used in the present study. The participants were to use an endogenous cue $(70 \%$ valid) to detect visually faint faces that appeared either on the left or the right side of fixation. It was shown that target detection performance improved with higher reward, without an increase in false alarm rates.

However, reward can also have an adverse effect on behavioral performance. Recent research has shown that previous reward history could be detrimental if a reward had been associated with a stimulus feature, such as a color. In comparison with studies that had rewarded participants on the basis of their overall performance in a given task, these studies associated a particular feature with reward, and through this association, attention was involuntarily attracted to the stimulus. A previously rewarded color was shown to be detrimental to performance if the color used as a distractor in a subsequent task (Anderson, Laurent, \& Yantis, 2011). Naming the color of a word during incongruent trials in a Stroop task is even harder if the meaning of the word is semantically associated with a previously rewarded color (Krebs, Boehler, \& Woldorff, 2010). This involuntary attentional capture by stimulus-reward association is distinct from conventional stimulus- or goal-driven attention, in that the attentional system favors previously reward-associated stimuli over other, more perceptually salient items and under explicit instructions to ignore the stimuli. This stimulus-reward association has been shown to persist even after seven months without any retraining in the interim period and when the participants could not recall the previously learned stimulus-reward associations consciously (Anderson \& Yantis, 2013), even when the previously rewarded stimulus was associated with a penalty in the subsequent task (Camara, Manohar, \& Husain, 2013). Anderson and colleagues (2011) proposed that a mechanism exists in which lasting representations of learned stimulus-reward relationships independently exert an influence on selective attention. They termed this mechanism value-driven attentional selection, in which stimuli that have been previously associated with reward through learning involuntarily capture attention, even when they are entirely task-irrelevant and when rewards are no longer available.

Interestingly, some evidence suggests that this adverse involuntary aftereffect of reward can be overcome if the inhibitory response is rewarded. Using a go/no-go task, Sinopoli, Schachar, and Dennis (2011) showed that withholding a response during a no-go trial, which is usually challenging, improved if participants were rewarded for correctly withholding the response. Boehler, Hopf, Stoppel, and Krebs (2012) similarly attested to this by showing that cancellation of a motor response is accelerated by reward: Participants showed a higher stop rate for stop signals in the middle of a motor response if the stop performance was related to a reward.
The aim of the present study was to extend the previous finding that an adverse involuntary aftereffect of reward can be overcome by rewarding a counterresponse. Specifically, we examined whether or not strategic control can be employed to prevail over an involuntary attentional bias for a previously reward-associated stimulus by associating a bigger reward with a different stimulus. In this study we used financial incentives to motivate participants, because such rewards have proven to be an effective means of enhancing participants' motivation in behavioral experiments (Pessoa \& Engelmann, 2010). Typically, participants are advised at the start of the experiment that a specific amount of money will be awarded for responses that meet predefined parameters of speed and accuracy. Here we offered participants varying amounts of monetary incentives for the most accurate and fastest response on average. It was expected that such an incentive would lead to improved performance, and that the best performance would be observed in conditions offering potential for the highest reward.

The experimental design of the present study was based on the Posner cueing task (Posner, 1980). The letter cues X and T were used to indicate the likely location of the target. The targets were either vertical or horizontal line segments, and participants were instructed to discriminate the target by pressing corresponding keys. It was expected that when the target appeared at the cued location (i.e., the cue was valid), responses would be faster and more accurate than when the target appeared at the uncued location (i.e., the cue was invalid).

Our study involved two phases. For the first phase, targets were associated with one cue (X), and for the second phase, targets were associated with a different cue (T). Correct responses were rewarded with points. In order to ascertain the effect of these incentives, participants were randomly allocated to one of four groups. Each participant was involved in two phases of the experiment and experienced one of the reward contingencies outlined in Table 1.

During reward phases, a prize was rewarded to those who achieved the most points and the fastest average RT. In the study, we aimed to determine whether the incentive of points and a prize would motivate participants to change their responses to the cues.

The first experimental condition (reward-no reward) was designed to show attentional bias toward a previously rewardassociated cue. As such, we hypothesized that attentional bias toward the previously rewarded letter cue $\mathrm{X}$ would be observed in Phase 2. In the second experimental condition

\begin{tabular}{llll}
\cline { 2 - 4 } $\begin{array}{l}\text { Table 1 Reward } \\
\text { contingencies } \\
\text { experienced by each } \\
\text { group }\end{array}$ & Group & Phase 1 & Phase 2 \\
\cline { 2 - 4 } & 1 & Reward \$50 & No reward \\
& 2 & Reward \$50 & Reward \$100 \\
& 3 & No reward & Reward \$50 \\
& 4 & No reward & No reward \\
\hline
\end{tabular}


(reward-higher reward), we examined the effects of further incentives following previous reward history with the letter cue X. It was hypothesized that the attentional bias toward a currently rewarded cue $(\mathrm{T})$ would compete with the attentional bias toward the previously rewarded cue $(\mathrm{X})$, so that the attentional bias toward the cue letter $\mathrm{X}$ would be overcome by the availability of a bigger reward that was associated with the cue letter $\mathrm{T}$. The third experimental group (no reward-reward) served as a control group. We hypothesized that for this group, no effect would be observed in Phase 2 from the previous phase, since no reward was associated with the previous cue (i.e., faster responses on valid than on invalid trials in Phase 2). The fourth experimental group (no reward-no reward) served as a baseline. We hypothesized that performance would be comparatively worse for this group, with longer RTs being predicted for invalid than for valid trials in Phase 1, and longer RTs for invalid than for valid trials in Phase 2 (i.e., the standard cueing effect for both Phases 1 and 2). Of particular interest in the present study was the performance of the reward-higher reward group. For this cohort, one of two results was expected: The reward incentive of the second phase might surpass any cue-reward association established in the first phase of the experiment, or the cue-reward association established in the initial phase of the experiment might interfere with responses in the second phase.

\section{Method}

\section{Participants}

Forty-eight volunteers between 18 and 52 years of age $(M=$ 33.48 years) were recruited for this study. Of these, 25 were

Fig. 1 Sequence of events in Phase 1 , in which $X$ was the cue (top, valid; bottom, invalid). Phase 2 differed from Phase 1 only in that $\mathrm{T}$ was the cue, rather than $\mathrm{X}$ female and 23 male. Written consent was obtained from all participants, and all procedures were approved by the Charles Sturt University School of Psychology Ethics Committee. The majority $(77.1 \%)$ of participants were right-handed.

\section{Apparatus}

The experiment was conducted on a Dell Inspiron 17R computer controlled by an Intel Core i7 CPU. The screen resolution was $1,920 \times 1,080$. The Inquisit software was used to control the presentation and timing of visual stimuli. Participants viewed the monitor from a distance of approximately $60 \mathrm{~cm}$ in a quiet, dimly lit room. Each trial consisted of three displays: a fixation display, a letter cue display, and a target display (see Fig. 1).

For the fixation display, a white cross was presented in the middle of the screen against a black background. The visual angles subtended by each letter cue were $1^{\circ}$ (width) $\times 1.5^{\circ}$ (height). The distance between the mid-vertical line and the center of each letter cue was $6.5^{\circ}$, and the distance between the mid-horizontal line and the center of each letter cue was $3^{\circ}$ (i.e., the cue letters were presented slightly above the mid-horizontal line). The target was either a horizontal or a vertical line segment. The visual angles subtended were $2^{\circ}$ (width) $\times 0.2^{\circ}$ (height) for the horizontal line segment, and $0.2^{\circ}$ (width) $\times 2^{\circ}$ (height) for the vertical line segment. The distance between the center of the screen and the center of the target was $6.5^{\circ}$, and the target appeared on the same horizontal line as the fixation point.

\section{Procedure}

Participants were advised that they would see a central fixation cross and a set of two letters, $\mathrm{X}$ and T, on either side of the

\section{$\underline{\text { Valid Trial }}$}

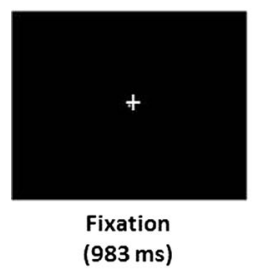

$\underline{\text { Invalid Trial }}$

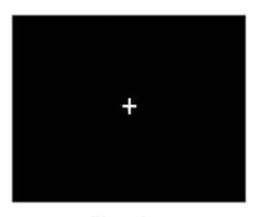

Fixation

(983 ms)
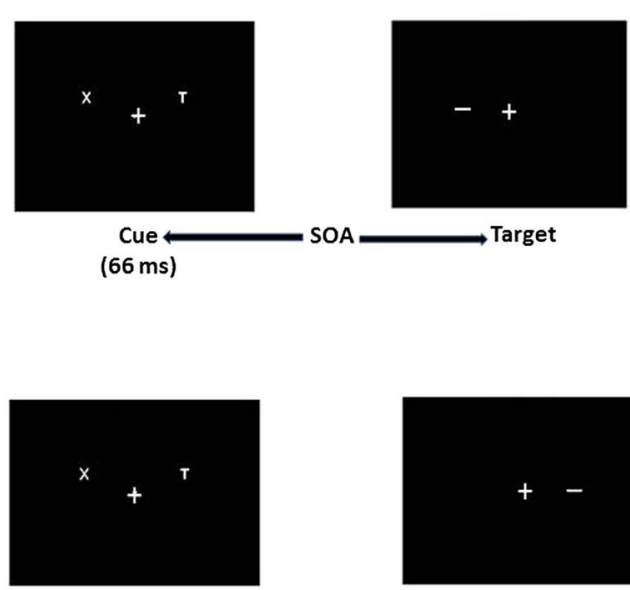

Cue $\rightleftarrows$ SOA $\longrightarrow$ Target (66 ms) 
fixation, followed by a target presented on either the left or the right side of the screen. Participants were instructed to fixate their eyes on the fixation cross. They were advised that the target would be either a horizontal or a vertical line segment, and they were instructed to press either the " $z$ " or the "." key, depending on the target's orientation. The corresponding response keys were counterbalanced.

The experiment was run in two phases of trials. For the first phase, participants were advised that the target would usually appear on the same side as the letter X, whilst for the second phase they were advised that it would usually appear on the same side as the letter $\mathrm{T}$. This aspect was emphasized by the instructor, recommending that participants use the cue to predict the probable location of the target.

The fixation cross appeared for $983 \mathrm{~ms}$, followed by the letters X and T, simultaneously for a period of $66 \mathrm{~ms}$. The time between cue onset and target onset (the stimulus onset asynchrony [SOA]) varied randomly and could be either $100 \mathrm{~ms}$ (SOA 1), $200 \mathrm{~ms}$ (SOA 2), or $500 \mathrm{~ms}$ (SOA 3). Trials could be either valid - that is, the target appeared on the same side as the cue - or invalid - it appeared on the opposite side from the cue. For each participant, $80 \%$ of the trials were valid, and the remaining $20 \%$ were invalid.

The target stimulus disappeared once participants had responded to it, as did the fixation cross. Depending on the response they had given, the participants were given performance feedback displayed on the screen: either "CORRECT" in green, with the points achieved below it (1 point for each correct response), or "WRONG" in red (no penalty for incorrect responses). After a pause of $1,700 \mathrm{~ms}$, the fixation cross would reappear, and the next trial commenced. Each participant experienced 16 practice trials, followed by 201 trials for Phase 1 and a further 201 trials for Phase 2. Each phase was further broken down into two different blocks (100 and 101 trials). Participants were allowed to take a break, if they wished, between blocks. At the end of each phase, their total scores were displayed on screen.

Participants were randomly allocated to one of the four groups, with 12 participants in each group (see Table 1 for the contingency experienced by each group). For rewarded phases, participants were advised that they would be rewarded a prize if they achieved the highest score in the fastest time for that group. Group 4 participants did not have an opportunity to receive a prize based on their performance in either Phase 1 or Phase 2. However, following completion of both phases, they were advised that they might be selected at random for a prize valued at $\$ 50$.

\section{Results}

A 4 (Group: 1, 2, 3, 4) $\times 2$ (Phase: 1, 2) $\times 2$ (Validity: invalid, valid $) \times 3$ (SOA: $100,200,500 \mathrm{~ms})$ mixed-design analysis of variance (ANOVA) was employed, with group as a betweensubjects variable and phase, validity, and SOA as withinsubjects variables. From the four-way mixed-design ANOVA, only interaction effects that included the group and phase variables were examined, since comparisons without these variables were not meaningful for the present study. Also, group comparisons in Phase 2 were carried out only between Groups 1 and 2 and Groups 3 and 4, because the performance during Phase 2 was contingent on Phase 1.

\section{Response times}

We found a moderate interaction between phase and group, $F(3,44)=2.4, p=.081, \eta_{\mathrm{p}}{ }^{2}=.141$. The mean RTs between different groups did not differ from each other for Phases 1 and 2 (all $p \mathrm{~s}>$.11). However, there were significant decreases in mean RTs from Phase 1 to Phase 2, by $48 \mathrm{~ms}$ for Group 1 ( $p$ $=.027), 92 \mathrm{~ms}$ for Group $2(p<.001)$, and $89 \mathrm{~ms}$ for Group 3 $(p<.001)$. The mean RTs for Phases 1 and 2 did not differ for Group $4(p=.21)$.

The interaction between phase, group, and validity was also significant, $F(3,44)=28.43, p<.001, \eta_{\mathrm{p}}{ }^{2}=.66$ (Fig. 2). For valid-versus-invalid trial comparisons, Group 1 responded significantly faster on valid than on invalid trials during Phase 1, by $222.5 \mathrm{~ms}$, but the reverse pattern was observed during Phase 2, in which the mean RT was faster for invalid trials than for valid trials by $218.7 \mathrm{~ms}$ (attentional bias for previously rewarded letter X, all $p \mathrm{~s}<.001$ ). Group 2 responded significantly faster on valid trials in comparison with invalid trials in both Phase 1 (by $296.3 \mathrm{~ms}$ ) and Phase 2 (by $391.5 \mathrm{~ms}$; intentional orienting toward letter $\mathrm{T}$ with a bigger reward) (all $p$ s $<.001$ ). Group 3 responded faster on valid than on invalid trials, by $199.6 \mathrm{~ms}$, only during Phase 2 (i.e., intentional orienting toward the reward-associated letter $\mathrm{T}$, all $p \mathrm{~s}<.001)$. No significant difference in RTs between valid and invalid trials was observed for Group $4(p>.104)$.

Continuing on with the three-way interaction, group comparisons for Phase 1 showed that the mean RTs did not differ between groups (all $p \mathrm{~s}=1$ ) on invalid trials. On valid trials, Group 2 was significantly faster than Group $3(p=.021)$ and Group $4(p=.06)$. During Phase 2, Group 2 was significantly slower than Group $1(p<.006)$ on invalid trials. On valid trials, Group 2 was faster than Group $1(p<.005)$. Phase comparisons showed that the mean RT decreased significantly from Phase 1 to Phase 2 on invalid trials, but increased on valid trials for Group 1 (attentional bias for the previously rewarded letter X, all $p$ s $<.001$ ). Groups 2 and 3 showed a significant decrease in RTs from Phase 1 to Phase 2 only on valid trials, but not on invalid trials (intentional orienting toward the reward-associated letter $\mathrm{T}$, all $p \mathrm{~s}<.001$ ). No significant difference was observed in the RTs between Phase 1 and Phase 2 for Group 4 on valid or invalid trials $(p>.284)$. 
$\mathbf{a}$

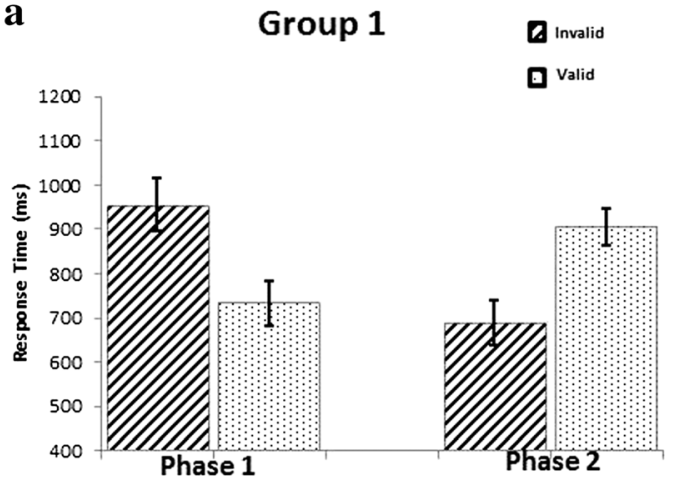

c

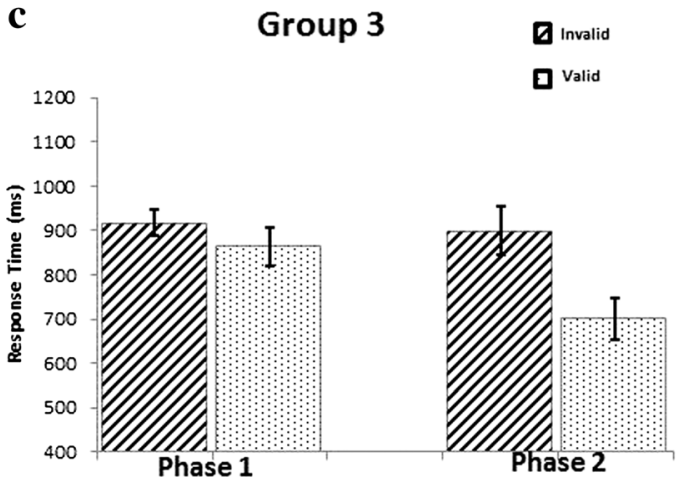

b

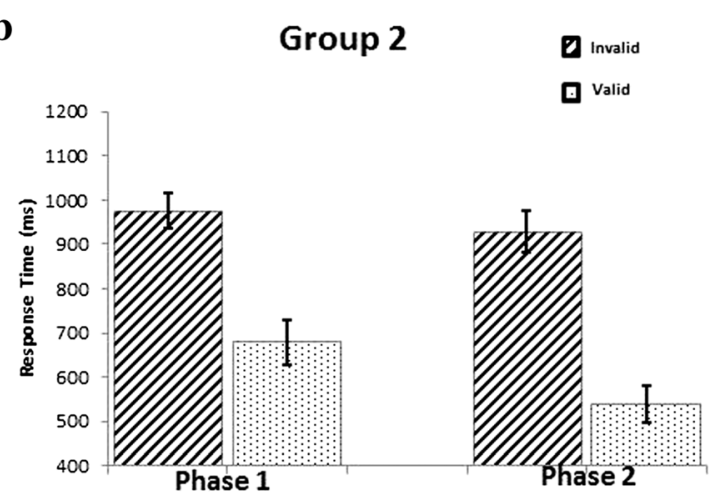

d

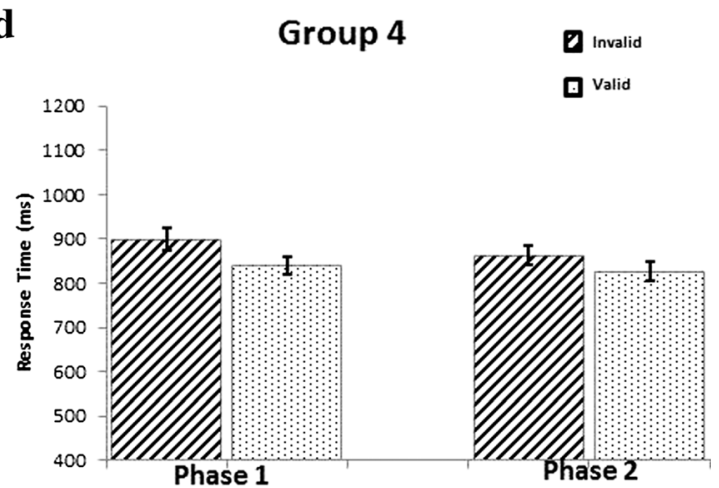

Fig. 2 Mean response times of each group on invalid and valid trials. Error bars indicate $95 \%$ confidence intervals (Cousineau, 2005)

Lastly, we observed a significant four-way interaction between phase, validity, SOA, and group, $F(6,88)=3, p=.01$, $\eta_{\mathrm{p}}{ }^{2}=.17$ (Figs. 3 and 4). Valid-versus-invalid trial comparisons revealed that Group 1 was faster on valid than on invalid trials at all SOAs during Phase 1 (all $p \mathrm{~s}<.001$ ). However, this trend was reversed during Phase 2, in which slower RTs were observed on valid than on invalid trials at all SOAs (all $p \mathrm{~s}<$ .001). In contrast, Group 2 showed faster RTs on valid than on invalid trials at all SOAs during both phases (all $p \mathrm{~s}<.001$ ). Group 3 showed faster RTs on valid than on invalid trials later, at SOA $500 \mathrm{~ms}$, during Phase $1(p=.014)$, whereas they responded faster on valid than on invalid trials at all SOAs during Phase 2 (all $p$ s $<.001$ ). Group 4 showed this effect only at SOA $200 \mathrm{~ms}$ during Phase $1(p=.052)$.

Continuing with the four-way interaction, group comparisons showed that no group difference was observed during Phase 1 at any of the SOAs on invalid trials. On valid trials, Group 2 was faster than Group 3 at SOA $200 \mathrm{~ms}(p=.019)$. Group 2 was also faster than Groups 3 and 4 at SOA $500 \mathrm{~ms}$ (all $p \mathrm{~s}<.018$ ). During Phase 2, Group 1 was faster than Group $2(p<.024)$ at the SOA of $100 \mathrm{~ms}$ on invalid trials. This was also the case at SOAs of 200 and $500 \mathrm{~ms}$, at which Group 1 was faster than Group 2 (all $p$ s $<.016$ ). On valid trials, Group 1 was slower than Group $2(p<.023)$. This trend continued to SOAs of 200 and $500 \mathrm{~ms}$, at which Group 1 was slower than Group 2 (all $p \mathrm{~s}<.027$ ). Phase comparisons revealed that
Group 1 showed a significant decrease in mean RTs from Phase 1 to Phase 2 at all SOAs on invalid trials, but a reverse pattern was observed for valid trials, in which a significant increase was observed from Phase 1 to Phase 2 (all $p \mathrm{~s}<$ $.001)$. For Groups 2 and 3, a decrease in mean RT from Phase 1 to Phase 2 was only shown for valid trials at all SOAs (all $p \mathrm{~s}$ $<.001)$, without any change for invalid trials. No change in mean RT from Phase 1 to Phase 2 was observed for Group 4 (all $p \mathrm{~s}>.233$ ). Lastly, SOA comparisons revealed that during Phase 1, Group 1 responded significantly faster at SOA $200 \mathrm{~ms}$ than at SOA $500 \mathrm{~ms}$ on invalid trials $(p=.025)$. On valid trials, they responded faster at SOA $200 \mathrm{~ms}$ and $500 \mathrm{~ms}$ than at SOA $100 \mathrm{~ms}(p<.001)$, and faster at SOA $500 \mathrm{~ms}$ than at SOA $200 \mathrm{~ms}(p=.004)$. During Phase 2, they responded faster at SOAs of 200 and $500 \mathrm{~ms}$ than at SOA $100 \mathrm{~ms}$ (all $p \mathrm{~s}$ $<.019$ ) on invalid trials, but not on valid trials. Group 2 showed difference in mean RTs for different SOAs only on valid trials, but not on invalid trials, for both phases: They responded faster at SOAs of 200 and $500 \mathrm{~ms}$ than at $100 \mathrm{~ms}$, and faster at SOA $500 \mathrm{~ms}$ than at SOA $200 \mathrm{~ms}$ (all $p \mathrm{~s}<.001)$. Similarly, Group 3 responded faster at SOA $500 \mathrm{~ms}$ than at SOA 100 or $200 \mathrm{~ms}(p \mathrm{~s}<.003)$ only on valid trials during Phase 1, and responded faster at SOAs of 200 and $500 \mathrm{~ms}$ than at SOA $100 \mathrm{~ms}$, and faster at SOA $500 \mathrm{~ms}$ than at SOA $200 \mathrm{~ms}$, only on valid trials during Phase 2 (all $p \mathrm{~s}<$ .022). Group 4 showed a similar trend: They responded faster 

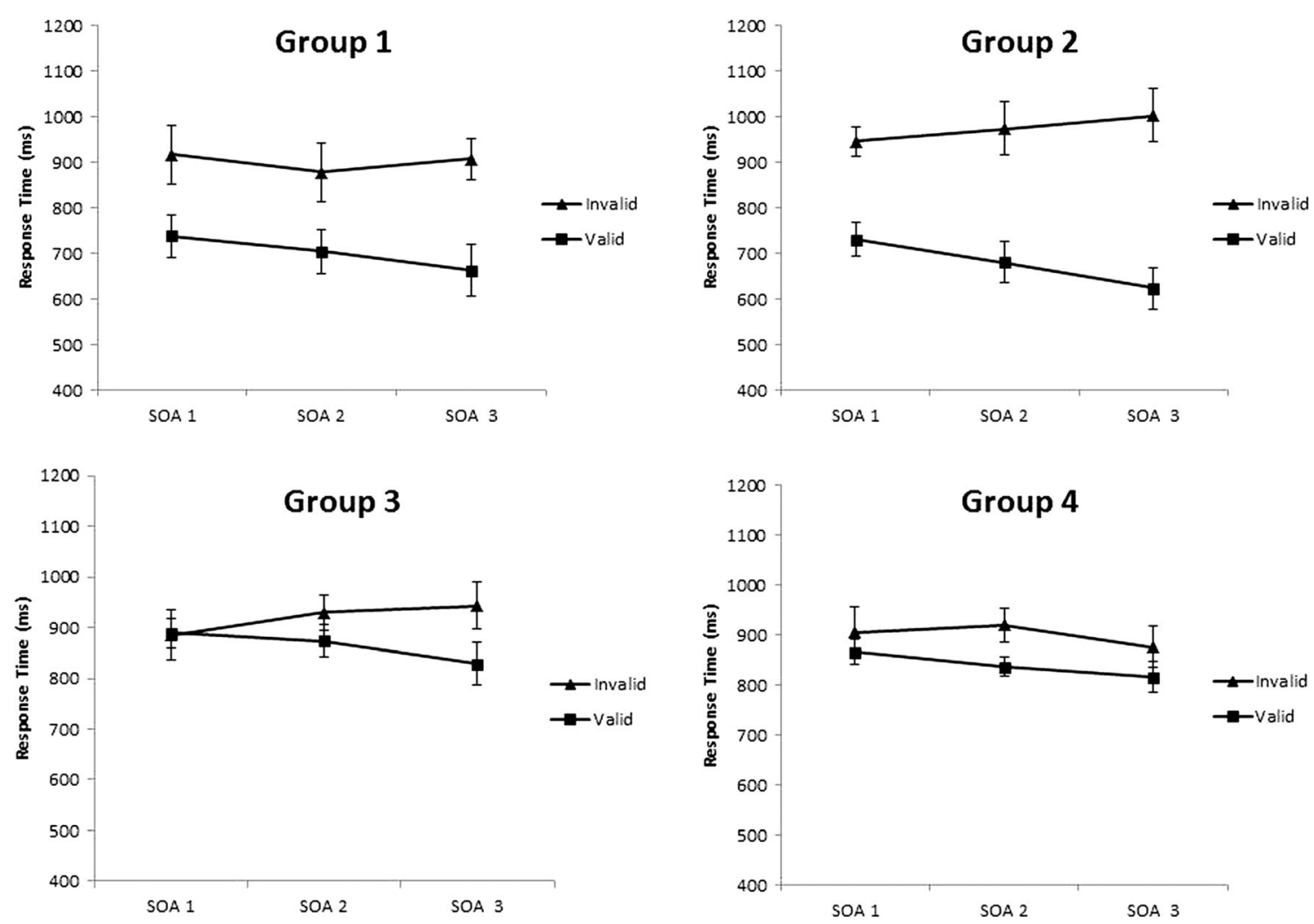

Fig. 3 Mean response times for each group at each stimulus onset asynchrony (SOA), Phase 1. Error bars indicate $95 \%$ confidence intervals (Cousineau, 2005)
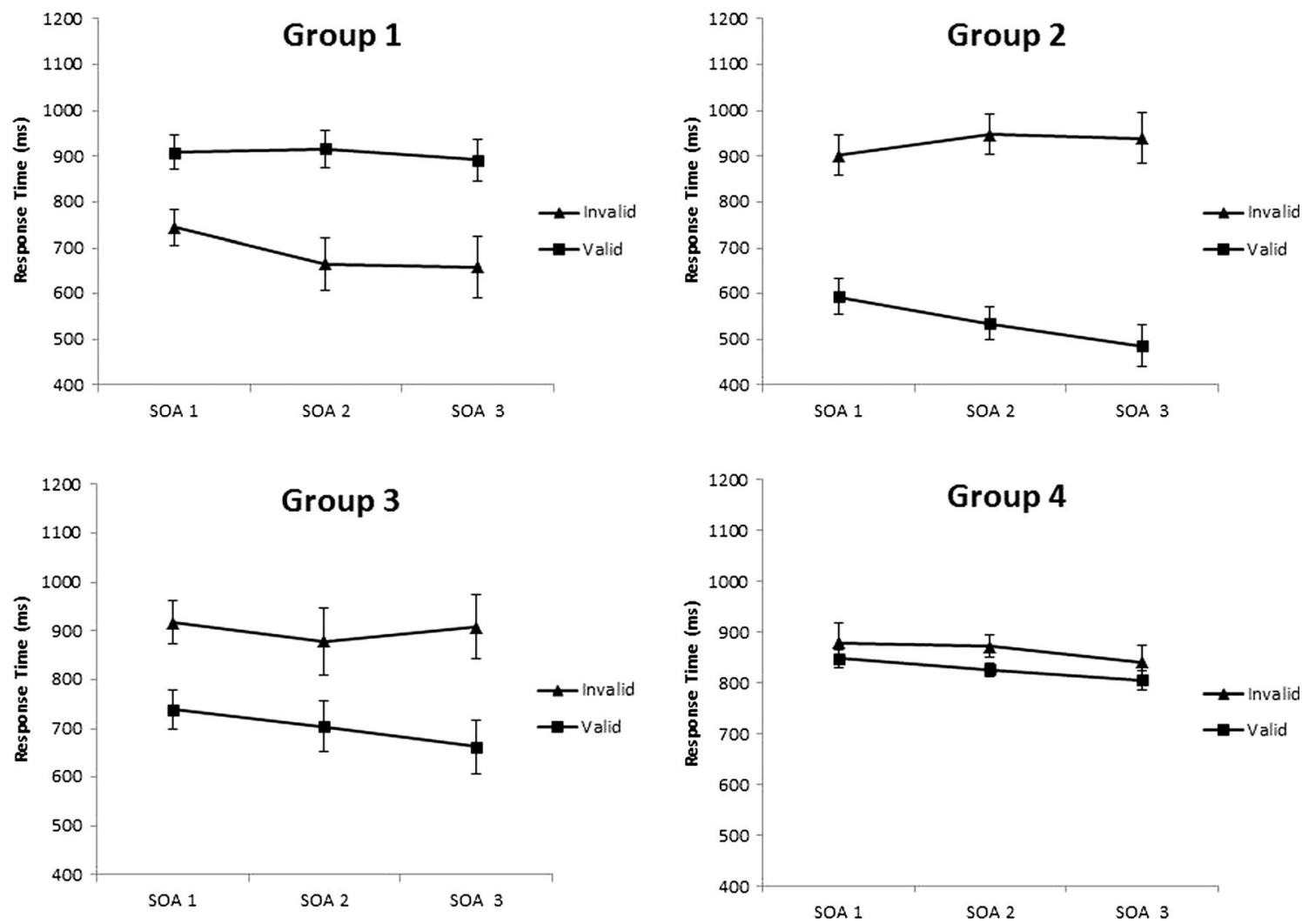

Fig. 4 Mean response times for each group at each stimulus onset asynchrony (SOA), Phase 2. Error bars indicate $95 \%$ confidence intervals (Cousineau, 2005) 
at SOAs of 200 and $500 \mathrm{~ms}$ than at SOA $100 \mathrm{~ms}$ only on valid trials for both phases (all $p \mathrm{~s}<.032$ ). No other interaction effects were significant.

\section{Accuracy}

The same 4 (Group: 1, 2, 3, 4) $\times 2$ (Phase: 1, 2) $\times 2$ (Validity: invalid, valid) $\times 3$ (SOA: 100, 200, $500 \mathrm{~ms}$ ) mixed-design ANOVA was performed for accuracy. No significant main or interaction effects were observed for any of the variables.

\section{Discussion}

Numerous studies have shown that reward contingencies have a significant influence on the inducement of motivation and the allocation of attention (Awh et al., 2012; Chelazzi et al., 2013; Hickey, Chelazzi, \& Theeuwes, 2010). With the present study, we aimed firstly to show this motivational effect of reward in the allocation of attention, and its attentional bias as a result, and secondly, to investigate whether or not motivating participants to strategically orient toward a different stimulus is effective in overcoming an attentional bias toward a previously rewarded stimulus. We examined a series of experimental conditions in which motivation was manipulated by varying the amount of performance-based financial reward. Four different groups of participants were subjected to a spatial-cueing task in which each experienced a different combination of reward and/or no-reward contingency, across two different phases, with an associated range of predictions regarding the effects of the contingency on performance. These predictions were supported by the results obtained.

Firstly, we hypothesized that an attentional bias would be observed toward previously valid cues when participants were initially offered an opportunity for a performance-based reward, and the effect (i.e., the bias toward the previously rewarded cue) would persist even when the strategy was no longer valid in Phase 2 of the task. An attentional bias toward the previously valid cues was demonstrated by an analysis of the performance of Group 1. Of particular interest was a comparison of the RTs on valid versus invalid trials in each phase. In Phase 1 of the experiment, Group 1 participants were given the incentive of a $\$ 50$ prize if they achieved the best performance. For this phase, it was expected that - congruent with previous research findings (e.g., Small et al., 2005)—performance would be optimized by the incentive. This was the case, with the \$50 incentive offered in Phase 1 inducing a cueing effect as early as at the SOA of $100 \mathrm{~ms}$. Phase 2 of the experiment involved a change of cue (from X to T), so that the cue from Phase 1 became the distractor (associated with invalid trials) and the previous distractor now became the cue (associated with valid trials). No performance-based incentive was offered in this phase. It was predicted that the cue from
Phase 1 would still bias the attention of participants toward the letter cue X, due to its reward history. This would be manifested as faster RTs on invalid than on valid trials. The results indicated that the previously valid cue letter X was still commanding participants' attention in Phase 2, causing a rapid response to invalid trials but a delayed response to valid trials at each SOA, as early as $100 \mathrm{~ms}$.

Given that they were provided with specific instructions between phases that the cue had changed and that no value was any longer associated with the original cue, we could assume that they aimed to attend to the new cue. Their failure to do so may indicate that the attentional bias toward the earlier cue was partly involuntary. Reward or expectations of receiving a reward are generally considered to be the impetus for all goal-directed actions, synonymous with motivation, and to be highly influential on the perceptual, attentional, and cognitive control processes that facilitate behavioral performance (Pessoa \& Engelmann, 2010; Small et al., 2005). Such evidence led us next to hypothesize that it might be possible to overcome the effects of attentional bias by the previously valid cue - the letter X-by offering one group of participants an incentive for correct responses in Phase 1 of the experiment (letter $\mathrm{X}$ as the valid cue), followed by a higher-value incentive in Phase 2 (letter $\mathrm{T}$ as the valid cue). The procedure for Group 2 was identical to that for Group 1 (i.e., the valid cue changed from $\mathrm{X}$ to $\mathrm{T}$ from Phase 1 to Phase 2), except that Group 2 were provided with a bigger incentive of $\$ 100$ for the best performance in Phase 2. When a cue associated with higher reward was available to motivate them, participants proved to be comparatively resistant to distraction caused by the previously rewarded but now irrelevant cue. This manifested as longer RTs on invalid than on valid trials for both Phases 1 and 2 .

Rather than being distracted by the previously rewarded cue, participants showed even larger validity effects in Phase 2 than in Phase 1 (Phase 2 - Phase $1=95.2 \mathrm{~ms}$ ). The strategic orienting toward the current cue that netted a greater reward competed with the attentional bias toward the previous cue that netted a significantly smaller reward, resulting in the bigger reward sufficiently motivating participants to direct attention to the cued location in Phase 2. Critically, this improvement in RTs was seen at an SOA as early as 100 ms. Overall responses for valid trials for this group were significantly faster than those for all other groups, and the observed improvements in speed did not result in accuracy trade-offs. This result supports the earlier findings that monetary rewards increase motivation and detection sensitivity during the orienting of attention (Pessoa \& Engelmann, 2010).

Group 3 participants underwent a procedure identical to those for Groups 1 and 2, but only in Phase 2 did they receive an opportunity for a reward, which was capped at $\$ 50$. We hypothesized that an incentive of $\$ 50$ would lead to faster responses for valid trials (with the letter cue T) in Phase 2 than for 
valid trials (with the letter cue $\mathrm{X}$ ) in Phase 1, in which correct responses did not result in reward. Each prediction was met, since Phase 2 elicited a cueing effect of $199.6 \mathrm{~ms}$, as compared to no effect in Phase 1. This indicates that simple exposure to cue-target associations does not result in a later attentional bias unless these associations are coupled with reward. For Group 4, there was no reward incentive in either phase of the experiment. We predicted that RTs would be longer for invalid than for valid trials in both Phases 1 and 2. However, Group 4 showed a cueing effect only during Phase 1 at an SOA of $200 \mathrm{~ms}$. Hence, it was concluded that an absence of reward resulted in static performance overall for this group.

We expected that the performance of Group 2-rapid and efficient use of valid cue in both Phase 1 and Phase 2-would differ markedly from that of Group 1 or 4 . This was the case, because Group 1 showed an attentional bias toward the previously rewarded cue and Group 4 exhibited static performance due to the absence of reward. Although no direct comparisons were undertaken between Groups 2 and 3 for Phase 2 (due to their differing reward histories in Phase 1), it was observed that Group 2's RTs were faster than those for Group 3, which was also rewarded in this phase but with the lesser value of $\$ 50$. The Group 2's performance was also significantly faster in Phase 2 than in Phase 1, in which a $\$ 50$ reward was on offer. These results are consistent with findings from previous studies that a stimulus associated with a higher reward has greater effects than a stimulus associated with a lower reward (Anderson et al., 2011; Veling \& Aarts, 2010). However, the present study is distinct from previous studies that had compared the strengths of attentional biases in a subsequent phase for participants who had been exposed to stimuli associated with high and low reward at the same time. The present results suggest that strategic orienting toward a high-reward cue competes with and surpasses the bias for a low-reward cue after the bias for the low-reward cue is acquired, rather than during the bias acquisition.

Considered together, these results support previous findings that reward associations play a direct and important role in motivating optimal performance on tasks that tax attentional resources. Emerging from the present study is the novel finding that attentional bias effects toward a previously valuable but now invalid cue can be eclipsed by strategic orienting to a different cue that is associated with rewards of greater value. Our findings imply that perhaps attentional bias toward a previously rewarded stimulus fundamentally represents a difficulty with overcoming or modifying a previous selection strategy, providing direct evidence that learned value influences attentional control in a way that may be mediated depending on ongoing goals.

Our findings are in direct contrast to those of Camara et al. (2013), who were able to demonstrate attentional bias by previously rewarded cues even when a new cue was associated with higher reward while the previously rewarded cue was simultaneously associated with a penalty. However, the present study differed notably from that of Camara and his colleagues in one important respect: Reward here was based on the overall task performance. The letter cues (X and $\mathrm{T})$ became associated with reward because they were useful in detecting and discriminating the targets faster and more accurately. Hence, the attentional bias toward the previously rewarded cue was formed in a more endogenous manner, whereas in Camara et al.'s study, reward was associated with a feature (a color) that attracted attention in a more exogenous fashion. The higher reward offered to Group 2 in Phase 2 produced larger validity effects than in the earlier phase, indicating that participants were more motivated to direct attention to the cued location. Interestingly, this improvement in RT was seen at an SOA of only $100 \mathrm{~ms}$, showing that the orienting of attention toward the reward was very rapid, in line with the neuroimaging studies of Kiss, Driver, and Eimer (2009) and Hillyard and Anllo-Vento (1998), who found an effect of reward in time frames of less than $200 \mathrm{~ms}$.

A recent study by Bucker and Theeuwes (2014) was able to show that in an exogenous task, rewards did not mediate initial orienting but were influential on reorienting at an SOA later than $170 \mathrm{~ms}$, which suggests that reward mediated attention in a top-down manner. Our findings highlighted clear effects of intentional orienting in response to rewarding cues-strategic orienting toward a cue with a bigger reward in Group 2 - that could be considered deliberate, since participants were forewarned of the potential reward available. However, the fact that the orienting effect toward valid cues was extremely rapid (at an SOA under $100 \mathrm{~ms}$ ) would mean that individuals were so adept at executing shifts in attention when motivated by reward that minimal conscious effort was required, and that processing was so efficient that RTs mirrored, or in fact were even faster than, those found on an exogenous task (see Bucker \& Theeuwes, 2014). It may be that these attentional biases are based on a mixture of exogenous and endogenous processes. These combined effects lend themselves better to recent theorizing by Ristic and Landry (2015) and Goodhew, Kendall, Ferber, and Pratt (2014), who have produced experimental results that imply that endogenous and exogenous controls of attention interact flexibly and are engaged jointly by a single behaviorally relevant cue, resulting in an additive effect that leads to increased sensory processing of attended targets.

Our findings are also largely congruent with appraisal theory (Pool, Brosch, Delplanque, \& Sander, 2014). Pool and colleagues found that devaluating an initially rewarded stimulus nullified the effects of value-driven attention capture, and related this to the affective relevance of the stimulus. Although our findings do not support the idea that this is entirely an involuntary effect, we could otherwise interpret our results within this framework by proposing that offering a higher reward in Phase 
2 for Group 2 had the effect of devaluing the initial reward and enhancing the affective relevance of the new cue. We posited that this reflects the possibility that attentional choices may in fact be made through a process whereby individuals orient and sequentially reject or accept the currently attended option, ultimately focusing on the highest subjectively valued opportunity. Anticipating a higher reward, individuals deliberately seek out the stimulus linked to the high reward and overlook the previously valued stimulus.

Affective relevance may also explain why our findings are at odds with those of Camara and colleagues (2013). Their study differed fundamentally from ours in that all of their participants received a reward according to their performance, whereas this study only offered the possibility of reward to those who performed the best in their group. As such, our study cannot be directly compared with theirs. However, as an approximate comparison it may be useful to consider that for their study they offered a maximum reward of $£ 15$ - which equates to approximately $\$ 30$ - whereas in our study the reward used to compete with attentional bias by the previously reward associated cue was relatively higher, at $\$ 100$. Perhaps the value of the reward used by Camara et al. was not subjectively valued highly enough by the participants to induce the motivation necessary to focus their attention on a novel target.

In summary, the present findings support previous work showing that under a high-reward motivational state, cognitive control mechanisms are recruited to a greater extent. In the present study we have shown that it is possible to eclipse the involuntary behavioral reactions elicited by a previously established reward contingency by amplifying the value of a different contingency. If a reward is appraised as being affectively relevant, motivation is enhanced and attention shifts rapidly toward the stimuli associated with it. However, the simultaneous presentation of another stimulus that is perceived as being more valuable results in the earlier stimulus being devalued and no longer being appraised as relevant. Subsequently, attention is no longer rapidly oriented toward the earlier stimulus. For this study, the effects cannot be described as purely reflexive because they were influenced by intentional control, although the shifts of attention observed were in time frames normally associated with the involuntary allocation of attention. However, in the experimental paradigm used, participants were given prior advice of the reward association, and we suggest that the improvement in performance was also substantially influenced by a conscious intention to orient attention as rapidly as possible, in anticipation of the reward at stake.

Our results are relevant to understanding environmental modulation of reward-directed behavior, and they may have implications for the treatment of pathologies characterized by attentional biases. On the basis of the present findings, heightened reactivity to cues that need to be ignored appears to be less the consequence of chancing upon salient cues in the course of one's everyday routine, and more an unavoidable consequence of extremely efficient cognitive processing that is permanently readied to rapidly notice stimuli in the environment that offer the possibility of reward. What our study implies is that the reactivity to stimuli related to addiction is not a case of accidental encounters with the cues triggering addiction-related behaviors, but rather a case of somewhat involuntary active searching for these cues, which may lead to dysfunctional problematic behaviors (e.g., craving or the consumption of a substance of abuse). Indeed, patients suffering from drug dependence have been shown to be significantly more affected by distractors previously associated with monetary reward than are healthy controls (Anderson, Faulkner, Rilee, Yantis, \& Marvel, 2013), suggesting that these patients are distracted not only by drug-related stimuli, but by stimuli associated with reward in general.

However, our results also suggest that affected individuals may be able to curb this tendency by focusing on more rewarding stimuli. Professionals enlisted to treat those suffering from pathologies characterized by maladaptive attentional biases could help these individuals focus on the role of selfefficacy and help them understand that it is within their conscious control to disengage from tendencies to seek out rewarding stimuli associated with dysfunctional behavior. Focusing on different rewards that are also affectively relevant to them, but less harmful, may prove beneficial in treatment.

Identifying what constitutes a more highly valued reward for a particular individual may present the greatest challenge, especially for those affected by addiction. Vulnerability to relapse has been attributed to the increased incentive salience of drug cues and the decreased salience of intrinsically rewarding stimuli (Robinson \& Berridge, 1993). Cognitive training regimens centered on restoring sensitivity to naturally rewarding stimuli may be effective in increasing responsiveness to natural rewards, thus counteracting the effects of addiction on reward neurocircuitry. Mindfulness-based interventions may offer some promise, since they are linked with enhanced attention regulation, by increasing the ability to engage, disengage, and shift attention efficiently from one object to another (Garland, Froeliger, \& Howard, 2014). Levinson, Stoll, Kindy, Merry, and Davidson (2014) have recently reported experimental findings that the mindfulness practice of breath counting leads to less attentional capture by distractors formerly paired with reward, in a study that used a group of experienced meditators as participants. These findings warrant future research into the effectiveness of such practices utilizing clinical populations.

The improvements in performance noted for Group 2 in Phase 2 suggest that it may be revealing to conduct a further study, identical to this one, with the addition of another group that experiences no reward in Phase 1 and a $\$ 100$ reward in Phase 2. This would allow us to ascertain whether the improvements in performance following Phase 1 were 
comparable, regardless of reward history, when a potential $\$ 100$ reward was on offer in Phase 2. Alternatively, considering that our findings were at odds with the persistence of value-driven attention capture, demonstrated in the study of Camara et al. (2013) despite the availability of higher rewards, it may be useful to conduct a similar study comparing groups of participants who experience the Group 2 condition (reward/ higher reward) but who have the potential to earn different amounts of reward in Phase 2, ranging from $\$ 50$ to $\$ 100$. This might allow us to determine whether attentional bias is as markedly reduced if the increase in incentive is only slight, or if the effect is only noticeable once the value of reward reaches a specific threshold that becomes subjectively important to the participant.

Author note This research was financially supported by the School of Psychology, Charles Sturt University. Our thanks to Associate Professor Jeffrey Hamm for providing comments on the manuscript.

\section{References}

Anderson, B., \& Yantis, S. (2013). Persistence of value-driven attentional capture. Journal of Experimental Psychology: Human Perception and Performance, 39, 6-9. doi:10.1037/a0030860

Anderson, B. A., Laurent, P. A., \& Yantis, S. (2011). Value-driven attentional capture. Proceedings of the National Academy of Sciences, 108, 10367-10371. doi:10.1073/pnas.1104047108

Anderson, B. A., Faulkner, M. L., Rilee, J. J., Yantis, S., \& Marvel, C. L. (2013). Attentional bias for nondrug reward is magnified in addiction. Experimental and Clinical Psychopharmacology, 21, 499-506.

Awh, E., Belopolsky, A. V., \& Theeuwes, J. (2012). Top-down versus bottom-up attentional control: A failed theoretical dichotomy. Trends in Cognitive Sciences, 16, 437-443. doi:10.1016/j.tics. 2012.06.010

Bijleveld, E., Custers, R., \& Aarts, H. (2010). Unconscious reward cues increase invested effort, but do not change speed-accuracy tradeoffs. Cognition, 115, 330-335.

Boehler, C. N., Hopf, J. M., Stoppel, C. M., \& Krebs, R. M. (2012). Motivating inhibition-Reward prospect speeds up response cancellation. Cognition, 125, 498-503. doi:10.1016/j.cognition.2012. 07.018

Bucker, B., \& Theeuwes, J. (2014). The effect of reward on orienting and reorienting in exogenous cuing. Cognitive, Affective, \& Behavioral Neuroscience, 14, 635-646. doi:10.3758/s13415-014-0278-7

Camara, E., Manohar, S., \& Husain, M. (2013). Past rewards capture spatial attention and action choices. Experimental Brain Research, 230, 291-300. doi:10.1007/s00221-013-3654-6

Chelazzi, L., Perlato, A., Santandrea, E., \& Della Libera, C. (2013). Rewards teach visual selective attention. Vision Research, 85, 5872. doi:10.1016/j.visres.2012.12.005

Cousineau, D. (2005). Confidence intervals in within-subject designs: A simpler solution to Loftus and Masson's method. Tutorials in Quantitative Methods for Psychology, 1, 42-45.

Engelmann, J., Damaraju, E., Padmala, S., \& Pessoa, L. (2009). Combined effects of attention and motivation on visual task performance: Transient and sustained motivational effects. Frontiers in Human Neuroscience, 3, 4. doi:10.3389/neuro.09.004.2009

Gable, P. A., \& Harmon-Jones, E. (2008). Approach-motivated positive affect reduces breadth of attention. Psychological Science, 19, 476482. doi:10.1111/j.1467-9280.2008.02112.x
Garland, E. L., Froeliger, B., \& Howard, M. O. (2014). Mindfulness training targets neurocognitive mechanisms of addiction at the attention-appraisal-emotion interface. Frontiers in Psychiatry, 4, 173. doi:10.3389/fpsyt.2013.00173

Goodhew, S. C., Kendall, W., Ferber, S., \& Pratt, J. (2014). Setting semantics: Conceptual set can determine the physical properties that capture attention. Attention, Perception, \& Psychophysics, 76, 1577-1589. doi:10.3758/s13414-014-0686-3

Gray, J. (2001). Emotional modulation of cognitive control: Approachwithdrawal states double-dissociate spatial from verbal two-back task performance. Journal of Experimental Psychology: General, 130, 436-452.

Hickey, C., Chelazzi, L., \& Theeuwes, J. (2010). Reward changes salience in human vision via the anterior cingulate. Journal of Neuroscience, 30, 11096-11103. doi:10.1523/jneurosci.1026-10. 2010

Hillyard, S. A., \& Anllo-Vento, L. (1998). Event-related brain potentials in the study of visual selective attention. Proceedings of the National Academy of Sciences, 95, 781-787. doi:10.1073/pnas.95.3.781

Kiss, M., Driver, J., \& Eimer, M. (2009). Reward priority of visual target singletons modulates event-related potential signatures of attentional selection. Psychological Science, 20, 245-251. doi:10.1111/j.14679280.2009.02281.x

Krebs, R., Boehler, C., \& Woldorff, M. (2010). The influence of reward associations on conflict processing in the Stroop task. Cognition, 117, 341-347. doi:10.1016/j.cognition.2010.08.018

Levinson, D. B., Stoll, E. L., Kindy, S. D., Merry, H. L., \& Davidson, R. J. (2014). A mind you can count on: Validating breath counting as a behavioral measure of mindfulness. Frontiers in Psychology, 5, 1202. doi:10.3389/fpsyg.2014.01202

Pessoa, L., \& Engelmann, J. B. (2010). Embedding reward signals into perception and cognition. Frontiers in Neuroscience, 4, 17. doi:10. 3389/fnins.2010.00017

Pool, E., Brosch, T., Delplanque, S., \& Sander, D. (2014). Where is the chocolate? Rapid spatial orienting toward stimuli associated with primary rewards. Cognition, 130, 348-359. doi:10.1016/j. cognition.2013.12.002

Posner, M. I. (1980). Orienting of attention. Quarterly Journal of Experimental Psychology, 32, 3-25. doi:10.1080/ 00335558008248231

Ristic, J., \& Landry, M. (2015). Combining attention: A novel way of conceptualizing the links between attention, sensory processing, and behavior. Attention, Perception, \& Psychophysics, 77, 36-49. doi: 10.3758/s13414-014-0737-9

Robinson, T. E., \& Berridge, K. C. (1993). The neural basis of drug craving-An incentive-sensitization theory of addiction. Brain Research Reviews, 18, 247-291. doi:10.1016/0165-0173(93) 90013-p

Savine, A. C., Beck, S. M., Edwards, B. G., Chiew, K. S., \& Braver, T. S. (2010). Enhancement of cognitive control by approach and avoidance motivational states. Cognition and Emotion, 24, 338-356. doi: $10.1080 / 02699930903381564$

Sinopoli, K. J., Schachar, R., \& Dennis, M. (2011). Reward improves cancellation and restraint inhibition across childhood and adolescence. Developmental Psychology, 47, 1479-1489. doi:10.1037/ a0024440

Small, D. M., Gitelman, D., Simmons, K., Bloise, S. M., Parrish, T., \& Mesulam, M. M. (2005). Monetary incentives enhance processing in brain regions mediating top-down control of attention. Cerebral Cortex, 15, 1855-1865. doi:10.1093/cercor/bhi063

Veling, H., \& Aarts, H. (2010). Cueing task goals and earning money: Relatively high monetary rewards reduce failures to act on goals in a Stroop task. Motivation and Emotion, 34, 184-190.

Verbruggen, F., \& De Houwer, J. (2007). Do emotional stimuli interfere with response inhibition? evidence from the stop signal paradigm. Cognition and Emotion, 21, 391-403. 